\title{
6. Verschärfung der Dokumentationsanforderungen von steuerinduzierten Lenkpreisen als Zukunftstrend
}

Zukünftig wird auch aufgrund von krisenbedingten Defiziten in vielen Ländern mit weiteren Verschärfungen bei der steuerrechtlichen Angemessenheitsprüfung von Verrechnungspreisen gerechnet. ${ }^{401}$ Unternehmen müssen aufgrund von international erwarteten Anforderungsreformen an die Verrechnungspreisdokumentation durch die OECD und die Einführung einer länderbezogene Berichtserstattung, dem sog. "Country-by-Country Reporting", mit erheblichen Neuerungen und höherem Ressourcenaufwand rechnen. ${ }^{402}$ Die abteilungsübergreifende Zusammenarbeit wie von der Steuerabteilung und dem Controlling ist hierbei wesentlich, um konsistente sowie zeitgerechte Dokumentationen zu erstellen. Mit den Dokumentationsvorschriften für Verrechnungspreise werden häufig anknüpfende Sanktionsmaßnahmen bei Missachtung dieser gesetzlich normiert. ${ }^{403}$ Bei nicht sorgfältiger Dokumentation besteht ein erhöhtes Risiko der Einstufung des Verrechnungspreises als nicht angemessen. ${ }^{404}$ Daher ist eine widerspruchsfreie Dokumentation, die die Informationsasymmetrien zwischen Unternehmen und Steuerbehörde auflösen, zu verkürzten Steuerprüfungen und zur Minimierung von Korrekturrisiken führen kann, international von hoher Bedeutung.

Da die Abdeckung von bestehenden Risiken von Verrechnungspreisen bisher in der Unternehmenspraxis als unzureichend empfunden wird und oft zu Spannungen zwischen dem Unternehmen und Steuerbehörde führt, ${ }^{405}$ wird eine Erweiterungsmöglichkeit dieser Simulationsstudie in der Integration weiterer bzw. branchenspezifischer Risiken gesehen. Ein Beispiel hierfür wäre die Modellerweiterung um einen Risikofaktor für riskante spezifische Investitionsentscheidungen.

\footnotetext{
${ }^{401}$ vgl. Baginova/Thomasberger/Wipfler [Unternehmenspraxis] 20 und Hanken/Kleinhietpaß [Spannungsfeld] 447

$402 \mathrm{vgl}$. auch folgend Bärsch/Engelen/Färber [Dokumentation] 972 und $980 \mathrm{ff}$.

${ }^{403} \mathrm{vgl}$. Becker [Gewinnabgrenzung] 60, Rosar [Dokumentation] 179 und Joecks/Kaminski [Sanktionsvorschriften] $65 \mathrm{ff}$.

$404 \mathrm{vgl}$. auch folgend Roller [Dokumentation] $326 \mathrm{ff}$.

${ }^{405} \mathrm{vgl}$. Hervé/Ackerman/Stock [Risiko] 619
} 